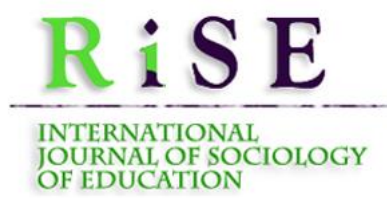

Instructions for authors, subscriptions and further details:

http://rise.hipatiapress.com

\title{
El Sentimiento de Pertenencia de los Jóvenes Chinos de la Segunda Generación de la Comunidad de Madrid
}

Weina Zhou ${ }^{1}$

Jesús Miguel Rodríguez-Mantilla ${ }^{1}$

1) Complutense University of Madrid, Spain

Date of publication: February $25^{\text {th }}, 2020$

Edition period: February 2020-June 2020

To cite this article: Zhou, W. \& Rodríguez-Mantilla, J.M. (2020). El Sentimiento de Pertenencia de los Jóvenes Chinos de la Segunda Generación de la Comunidad de Madrid, International Journal of Sociology of Education, 9(1), 34-59. doi: 10.17583/rise.2020.4622

To link this article: http://dx.doi.org/10.17583/rise.2020.4622

PLEASE SCROLL DOWN FOR ARTICLE

The terms and conditions of use are related to the Open Journal System and to Creative Commons Attribution License (CC-BY) 


\section{The Sense of Belonging of the Young Chinese of the Second Generation of the Community of Madrid}

Weina Zhou

Complutense University of Madrid
Jesús Miguel Rodríguez-Mantilla

Complutense University of Madrid

(Received: 2 September 2019; Accepted: 3 January 2020; Published: 25 February 2020)

\section{Abstract}

It is relevant to know the factors that determine the sense of belonging to understand the importance of this feeling from its different contents of identification, affective bonding and construction of meanings with the group. The aim of this study is to analyze the feeling of belonging of the second-generation Chinese young people residing in the Community of Madrid. To this end, a non-experimental study has been carried out, applying a questionnaire that allowed determining the cognitiveaffective and social characteristics of the subjects, with a total sample of 205 people. The possible differences in the feeling of belonging were analyzed according to sociodemographic variables (age, sex, country of birth, educational level, etc.). The results show that the subjects have, in general terms, a feeling of positive belonging. It is appreciated that most of the subjects feel a strong union towards the ethnic group to which they belong, they do not feel rejected by the Spanish people or of other ethnic groups and believe that respect for the customs and traditions of both countries is important, as well as the norms and good behaviors to be accepted by society.

Keywords: feeling of belonging, second generation, young Chinese, ethnic group 


\title{
EI Sentimiento de Pertenencia de los Jóvenes Chinos de la Segunda Generación de la Comunidad de Madrid
}

\author{
Weina Zhou \\ Jesús Miguel Rodríguez-Mantilla \\ Complutense University of Madrid \\ Complutense University of Madrid
}

(Recibido: 2 Septiembre 2019; Aceptado: 3 Enero 2020; Publicado: 25

Febrero 2020)

\section{Resumen}

Es relevante conocer los factores que determinan el sentido de pertenencia para entender el significado de este sentimiento a partir de sus diversos temas de identificación, relación afectiva y creación de la identidad en el grupo. El presente trabajo tiene como objetivo fundamental analizar el sentimiento de pertenencia que tienen los jóvenes chinos de la segunda generación que residen en la Comunidad de Madrid. Para ello, se ha realizado un estudio de tipo no experimental, aplicando un cuestionario que permitió determinar cuáles son las características cognitivoafectivas y sociales de los sujetos, con una muestra total de 205 personas. Se analizaron las posibles diferencias en el sentimiento de pertenencia en función de variables sociodemográficas (edad, sexo, país de nacimiento, nivel educativo, etc.). Los resultados obtenidos muestran que los sujetos tienen, en términos generales, un sentimiento de pertenencia positivo. Se aprecia que la mayoría de los sujetos sienten una fuerte unión hacia el grupo étnico al que pertenecen, no se sienten rechazados por la gente española o de otros grupos étnicos y creen que es importante el respeto hacia las costumbres y tradiciones de ambos países, al igual que las normas y las buenas conductas para que sean aceptados por la sociedad.

Palabras clave: sentimiento de pertenencia, segunda generación, jóvenes chinos, grupo étnico 


\section{Zhou \& Rodríguez-Mantilla-Pertenencia Jóvenes Chinos}

$\mathrm{E}$

1 aumento de los grandes movimientos migratorios que acontecen globalmente durante las últimas décadas, ha incrementado el interés por el estudio del desarrollo de la identidad, principalmente en inmigrantes y grupos minoritarios (Arenas \& Urzúa, 2016; Clemente, Espinosa \& Antón, 2013). La temática de la identidad y la educación se va a reforzar como un espacio para la práctica y la investigación de la pluriculturalidad, porque es lo que sirve de referencia a los jóvenes para incorporarse al modelo de las sociedades de acogida. En este sentido, Vega (2011) afirma que:

Debemos concentrarnos en elaborar herramientas de investigación que nos permitan reflejar estas dinámicas en nuestros trabajos de campo, pensar que la integración no es posible sin el reconocimiento de la diversidad, sin el reconocimiento de las nuevas identidades y sin el cambio de los modelos educativos homogeneizantes. (p.1647)

En España, al igual que sucede a nivel internacional, la población inmigrante ha aumentado significativamente durante los últimos años. Según refleja el Avance de la Estadística del Padrón Continuo a 22 de enero de 2019 publicado por el Instituto Nacional de Estadística (INE, 2019), el número de personas extranjera en el territorio español se sitúa en 4.734.691, de los cuales 215.748 personas pertenecen a la población China. España es, por tanto, uno de los principales destinos para los inmigrantes chinos en la sociedad actual.

Durante los últimos 40 años de reforma y apertura, un gran número de nuevos inmigrantes chinos han desembarcado en España. Con el rápido crecimiento de la población china en España, se han ido publicando numerosas investigaciones sobre dicha temática. Así por ejemplo, es posible encontrar estudios que analizan la situación actual de los inmigrantes chinos en España (Antolín, 2010; Robles-Llana, 2018; Rodríguez-Wangüemert, Rodríguez-Breijo \& Pestano-Rodríguez, 2019; Torruella, 2017), el rol de las mujeres chinas en España desde la perspectiva de género (Gutiérrez Sastre, 2014; Pérez, 2016; Sáiz López, 2015) o investigaciones sobre la educación de los inmigrantes chinos en el sistema educativo español (Huang, 2015; Wang \& Lorenzo, 2019). De igual modo, la literatura especializada pone de manifiesto que hay numerosas investigaciones sobre el sentimiento de 
pertenencia de grupos minoritarios inmigrantes (Barquín, 2009; Maillard, Ochoa y Valdivia, 2018; Merenstein, 2001; Terrén, 2011), pero son escasas las investigaciones sobre el sentimiento de pertenencia de la población inmigrante en España, y más concretamente sobre jóvenes chinos. Es por ello que, resulta de gran relevancia indagar sobre esta temática en un grupo étnico que está presente cada vez más en la sociedad española.

Esta presencia de la población china en España ha provocado, en gran medida, la existencia de una segunda generación de niños chinos nacidos en España, escolarizados en el sistema educativo español junto al resto de la población y dispersos por toda la geografía nacional (Olivencia, 2011; Hayes, 2013), si bien también son denominados como segunda generación a los niños nacidos en China que vienen a España a distintas edades y son escolarizados a su llegada, teniendo dificultades para integrarse con la población española. En este mismo sentido, y en el caso de los adolescentes que se incorporan como "nuevos" a la escuela en España, estos presentan mayores dificultades de integración y asimilación (Nieto, 2003), debido, por un lado, a las carencias que posee el sistema escolar en relación con la escolarización de extranjeros, pues existen pocos maestros de refuerzo que cubran las dificultades como el aprendizaje de la lengua, y tampoco hay profesores cualificados al respecto (Etxeberría \& Elosegui, 2010). Por otro lado, no cabe duda que a estas edades, los adolescentes necesitan mucho más tiempo para adaptarse y controlar de forma afectiva el sistema de escolarización que niños de poca edad, debido a cuestiones propias del momento evolutivo en el que se encuentran (Aparicio y Portes, 2014).

En este contexto, cobra especial relevancia el concepto de transnacionalismo el cual hace referencia a los vínculos que continúan existiendo con el lugar de procedencia a pesar de no encontrarse en él, destacando además la multiplicidad de tópicos existentes de la población china en España (Antolín, 2010). Los jóvenes de la segunda generación luchan por cambiar la imagen del resto de la población acerca de su estilo de vida. Se trata de tópicos que con el paso del tiempo quedarán diluidos en las sociedades modernas. Actualmente, cada vez más jóvenes chinos se alejan del modelo tradicional de su país de origen, dando lugar a un nuevo perfil, el de universitario, emprendedor, culto y hasta con posgrados y doctorados en las universidades españolas (Aparicio y Portes, 2014).

De este modo, el presente estudio resulta de gran relevancia en el ámbito 
del análisis del sentimiento de pertenencia, entendida éste como el conjunto de valores, percepciones, acciones y necesidades que constituyen un campo de comunicación y de interacción entre los individuos de una misma colectividad (Fenster, 2005), ya que cada vez es más habitual que aparezcan nuevos sectores de negocios encabezados o dirigidos por ciudadanos de origen chino, que en su día emigraron de China por diversos motivos o causas y que finalmente decidieron instalarse y crear su unidad familiar en un país diferente al que pertenecen. Por ello, resulta importante plantearse las siguientes cuestiones: ¿Qué ocurre con la segunda generación de chinos en la Comunidad de Madrid? ¿Cómo se sienten culturalmente los hijos de los que un día decidieron emprender su viaje a otro país con diferentes costumbres?

Brea (2014) afirma que es conveniente conocer los factores que determinan el sentido de pertenencia para entender la importancia de este sentimiento a partir de los diversos contextos de identificación, dimensión afectiva y elaboración de la identidad con el grupo y con el ambiente físico. Esta autora también señala que el sentido de pertenencia establece en sí mismo un elemento de la identidad personal, de los intereses, de las afiliaciones y del desarrollo humano, caracterizadas por los componentes cognitivos, afectivos y sociales.

Concretamente, y en relación con el componente Cognitivo del sentimiento de pertenencia, son diversas las investigaciones que remarcan la importancia de la identificación al grupo étnico al que pertenece el individuo, entendida como la etnicidad de la persona, caracterizado por el conocimiento de la historia y los valores del grupo étnico (Bartolomé, Cabrera, Espín, Del Campo, Marín, Rodríguez y Sabariego, 2000; MielesBarrera y Vesga, 2010). En esta misma línea, Smith Castro (2002) señala la necesidad que tiene el individuo de utilizar identificaciones étnicas para la categorización en su grupo de identidad, que no sólo valen como marco de referencia para estructurar la información del mundo social, sino también para proporcionar al individuo del conocimiento sobre lo que él es como persona. Por otro lado, autores como Cameron (2004), Strayhorn (2012) y Vargas Alfaro (2002) marcan la importancia que tiene las relaciones sociales dentro del sentido de pertenencia, pues la información que cada individuo tiene sobre el grupo étnico, será una respuesta afectiva y que las características del grupo darán forma a la identidad que influyen en la 
creación del sentimiento de pertenencia.

Por otro lado, el componente Afectivo resulta un elemento clave en el estudio de sentimientos de seguridad y apoyo con los patrones culturales del propio grupo étnico, sentimientos de las relaciones interpersonales y prioridad asociativa con individuos del propio grupo étnico en oposición a otros grupos étnicos (Brea, 2014). No obstante, estudios como los de Lara (2017) y Smith (2002) sobre las actitudes de jóvenes, han evaluado la etnicidad utilizando listas de adjetivos negativos, caracterizadas por el sentimiento de rechazo. Por lo tanto, una vez profundizado en el conocimiento de uno mismo y establecido una empatía y conocimiento de los demás a través de la interacción y las relaciones interpersonales, es posible iniciar un diálogo que trascienda el nivel cognitivo y afectivo conduciendo al individuo a posicionarse y actuar frente a cuestiones relacionadas con la discriminación (Bartolomé et al. 2000).

El contexto Social de los individuos es otro factor a tener en cuenta en el campo de estudio sobre el sentimiento de pertenencia. Molano (2007) señala que la identidad no es una noción fija, sino que se regenera de forma individual y colectiva y que se estimula continuamente de la influencia exterior, y Bartolomé et al. (2000) indican que la dimensión social está ligada al conocimiento y comprensión de las costumbres, normas y conductas que tienen implicaciones directas en el sentimiento de pertenencia del individuo. En este sentido, Lara y Martínez-Molina (2016) señalan en su estudio la importancia del factor de la exploración como un análisis de información y experiencias valiosas para la propia identidad. Asimismo, cada individuo es consciente de realizar una acción o mostrar una conducta determinada, derivada de una valoración introspectiva dentro de su propia escala de valores (Huerta Orozco, 2018).

La literatura especializada muestra que, generalmente, el uso de las identificaciones con el país o la cultura de origen dominan en la primera generación de inmigrantes, y es difícil que cambien durante toda su vida (Phinney, 2003). La segunda generación de inmigrantes se encuentra en una situación intermedia, entre su cultura de origen y la de acogida. Entre los inmigrantes de segunda generación, que ya han nacido en el país de acogida, es más probable que utilicen etiquetas compuestas, en las que ya incluyen referencias al país de acogida (Kiang, Perreira, \& Fuligni, 2011), es decir, poseer una doble identidad, entre el país de origen y el país de acogida. En 
diversas investigaciones se ha estudiado la relación de la identidad étnica con otros aspectos más allá del desarrollo evolutivo de la misma, como las relaciones familiares o el bienestar (Dávila de León y Jiménez García, 2014; Giró, 2010; Lara et al., 2016; Martínez, 2003; Terrén, 2011). De sus resultados se extrae que el contexto familiar es considerado una variable importante en el desarrollo de la identidad étnica.

Por todo ello, el objetivo principal del presente estudio es analizar el sentimiento de pertenencia que tienen los ciudadanos jóvenes chinos de la segunda generación, es decir, los hijos de los migrantes chinos que residen en la Comunidad de Madrid. De igual modo, se pretende analizar el conocimiento que tienen sobre su grupo étnico, así como las posibles diferencias existentes en su nivel de pertenencia en función de diversas variables (sexo, edad, país de nacimiento, etc.) e identificar distintos perfiles de sujetos en función de los niveles de sentimiento de pertenencia que presentan.

\section{Diseño \\ Diseño}

\section{Método}

El presente estudio responde a un diseño no experimental con un enfoque cuantitativo de tipo ex-post facto y de carácter descriptivo.

\section{Muestra}

Para este estudio se ha utilizado un procedimiento de muestreo de tipo no probabilístico e incidental, mediante la participación voluntaria de los sujetos, obteniendo una muestra final de 205 jóvenes chinos residentes en la Comunidad de Madrid. El 64,9\% son mujeres y el 35,1\% hombres. El 55,1\% de los participantes han nacido en España y el 41,5\% en China. El 67,8\% tienen edades comprendidas entre los 14 y los 24 años y el $32,2 \%$ restante entre los 25 y los 35 años. La distribución detallada de la muestra de estas y otras variables sociodemográficas que se utilizaron en el estudio aparece en la Tabla 1. 
Tabla 1.

Distribución de la muestra según variables sociodemográficas

\begin{tabular}{|c|c|c|c|}
\hline & & & Porcentaje \\
\hline \multirow{15}{*}{ 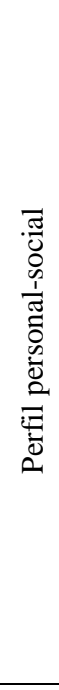 } & \multirow{2}{*}{ Sexo } & Mujer & 64,9 \\
\hline & & Hombre & 35,1 \\
\hline & \multirow{2}{*}{ Edad } & 14-24 años & 67,8 \\
\hline & & 25-35 años & 32,2 \\
\hline & \multirow{3}{*}{ País de nacimiento } & España & 55,1 \\
\hline & & China & 41,5 \\
\hline & & Otros & 3,4 \\
\hline & \multirow{4}{*}{$\begin{array}{l}\text { Tiempo de residencia en } \\
\text { España }\end{array}$} & 4 años o menos & 5,9 \\
\hline & & 5-10 años & 16,1 \\
\hline & & 11-19 años & 38,0 \\
\hline & & 20 años o más & 40,0 \\
\hline & \multirow{2}{*}{ Tienen amigos españoles } & No & 1,0 \\
\hline & & Sí & 99,0 \\
\hline & \multirow{2}{*}{ Tienen amigos chinos } & No & 2,9 \\
\hline & & Sí & 97,1 \\
\hline \multirow{8}{*}{ 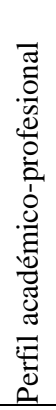 } & \multirow{5}{*}{ Nivel educativo } & Sin estudios & 1,0 \\
\hline & & Primaria & 0,5 \\
\hline & & Secundaria & 20,0 \\
\hline & & Formación Profesional & 8,3 \\
\hline & & Universitarios & 70,2 \\
\hline & \multirow{3}{*}{ Ocupación actual } & Estudiante & 57,1 \\
\hline & & Con empleo & 41,5 \\
\hline & & $\begin{array}{l}\text { No estudia ni busca } \\
\text { trabajo }\end{array}$ & 1,5 \\
\hline
\end{tabular}

\section{Instrumento}

Para alcanzar el objetivo de este estudio se ha utilizado un instrumento elaborado ad hoc, configurado por 24 ítems (ver Tabla 2), con una escala tipo Likert de 0 a 4, donde 0 indica Totalmente en desacuerdo y 4 Totalmente de acuerdo. La elaboración de la escala para evaluar el 
sentimiento de pertenencia de los jóvenes chinos se realizó mediante la configuración de un sistema de dimensiones, subdimensiones e ítems fundamentado en una amplia y profunda revisión teórica, asegurando así la validez de contenido del instrumento. De este modo, el cuestionario quedó estructurado en dos grandes dimensiones: Cognitivo-afectiva y Social.

Tabla 2.

Dimensiones, subdimensiones e ítems

\begin{tabular}{lll}
\hline DIMENSIÓN & SUBDIMENSIÓN & ÍTEMS \\
\hline \multirow{4}{*}{$\begin{array}{l}\text { COGNITIVO- } \\
\text { AFECTIVA }\end{array}$} & Conocimientos sobre el grupo de pertenencia & $1-4$ \\
\cline { 2 - 3 } & Sentimiento hacia el grupo étnico & $5-7$ \\
\cline { 2 - 3 } & Sentimiento de apoyo & $8-9$ \\
\cline { 2 - 3 } & Relaciones interpersonales & $10-14$ \\
\cline { 2 - 3 } SOCIAL & Sentimiento de rechazo & $15-17$ \\
\cline { 2 - 3 } & Costumbres y tradiciones & $18-19$ \\
\cline { 2 - 3 } & Normas & $20-22$ \\
\hline
\end{tabular}

Por un lado, la dimensión Cognitivo-afectiva está formada por cinco subdimensiones (con un total de 17 ítems): Conocimientos sobre el grupo de pertenencia (ítems 1-4 referidos al conocimiento sobre la cultura del grupo étnico), Sentimiento hacia el grupo étnico (ítems 5-7 aludiendo a las emociones hacia el grupo étnico), Sentimiento de apoyo (ítems 8-9 que comprenden la protección y defensa de otras personas), Relaciones interpersonales (ítems 10-14 entendidos como el vínculo de una interacción recíproca) y, por último, Sentimiento de rechazo (ítems 15-17 referidos a la sensación negativa de exclusión). Por su parte, la dimensión Social (constituida por 7 ítems) incluye las subdimensiones Costumbres $y$ tradiciones (ítems 18-19 que aluden al respeto hacia los hábitos de ambos países), Normas (ítems 20-22 entendidos como el conjunto de reglas de una comunidad para una mejor convivencia) y Conducta (ítems 23-24 referidos al comportamiento humano en la sociedad). La descripción de los ítems se especifica en las Tablas 3 y 4.

De igual modo, el cuestionario incluye 11 variables sociodemográficas, 
tales como: edad, género, país de nacimiento, tiempo de residencia en España, ocupación actual, nivel educativo, etc. (recogidos en la Tabla 1).

\section{Procedimiento de Recogida y Análisis de Datos}

Para llevar a cabo el presente estudio, en primer lugar, se realizó una búsqueda presencial de lugares donde hubiera más afluencia de población china, como puede ser en universidades, institutos, lugares de ocio, bazares o restaurantes chinos en la provincia de Madrid, especialmente en el barrio de Usera y en el Polígono Industrial Cobo Calleja de Fuenlabrada. Los sujetos que decidieron participar, respondieron al cuestionario que fue aplicado en formato papel y de forma online. La recogida de la información se llevó a cabo durante los meses marzo y abril de 2019.

Para el análisis de datos se utilizó el paquete estadístico SPSS 24. En primer lugar, se analizó la fiabilidad del instrumento de medida mediante el cálculo e interpretación del alfa de Cronbach. Posteriormente, se realizó un estudio descriptivo de cada uno de los ítems, junto a análisis diferenciales aplicando ANOVA de un factor y $t$ de student para muestras independientes, calculando el tamaño del efecto a través del estadístico eta cuadrado. Igualmente, se llevó a cabo un análisis clúster o de conglomerados por el método de $k$ medias para identificar distintos perfiles de sujetos en función de su nivel de sentimiento de pertenencia a su grupo étnico.

\section{Resultados}

\section{Fiabilidad del Instrumento}

Para el análisis de la fiabilidad del instrumento se calculó el Alfa de Cronbach, obteniendo un valor de 0,802 para el conjunto global de los ítems. De igual modo, se analizó la fiabilidad por dimensiones, obteniendo valores adecuados ( $\alpha$ dimensión Cognitivo-afectivo: $0,784, \alpha$ dimensión Social: 0,742).

\section{Análisis Descriptivos}

En términos globales, los sujetos muestran un nivel medio en la dimensión Cognitivo-afectiva, con un promedio general de 39,70 sobre 68 (ver Tabla 3). De forma específica, se observa que valoran con un nivel alto $l a$ 


\section{Zhou \& Rodríguez-Mantilla-Pertenencia Jóvenes Chinos}

comprensión de lo que significa formar parte de un grupo étnico, su sentimiento de felicidad, de orgullo y de una fuerte unión hacia su grupo étnico (ítems 1, 5, 6 y 7). La asistencia a organizaciones compuestas por personas del mismo grupo étnico (ítem 14) presenta una valoración de nivel medio, mientras que los sujetos manifiestan un nivel bajo del sentimiento de rechazo por parte de gente española, de discriminación por otros grupos étnicos y de recriminación por gente china por relacionarse con gente española (ítems 15, 16 y 17).

Tabla 3.

Medidas y desviaciones típicas de los ítems de la dimensión Cognitivo-afectiva

\begin{tabular}{llrl} 
& & Media & D.T. \\
\hline 1. & Comprendo lo que significa e implica formar parte de mi grupo étnico & 3,24 & 0,81 \\
2. & He hablado con otras personas para aprender más sobre los valores de mi grupo étnico 2,70 & 1,12 \\
3. & He invertido tiempo para averiguar más sobre la historia de mi grupo étnico & 2,47 & 1,15 \\
4. & Conozco más sobre la historia de China que de España & 1,60 & 1,35 \\
5. & Me siento feliz de ser miembro de mi grupo étnico & 3,26 & 0,94 \\
6. & Me siento orgulloso de mi grupo étnico al que pertenezco & 3,34 & 0,92 \\
7. & Siento una fuerte unión con mi propio grupo étnico & 2,94 & 1,00 \\
8. & Me siento apoyado por chinos cuando tengo problemas & 2,85 & 1,14 \\
9. & Me siento apoyado por españoles cuando tengo problemas & 2,40 & 1,10 \\
10. & Me siento mejor estando con personas chinas & 2,43 & 1,20 \\
11. Procuro relacionarme principalmente con chinos & 1,60 & 1,28 \\
12. Creo que es importante buscar y mantener relaciones con españoles & 2,83 & 1,02 \\
13. Prefiero que mi pareja sea de etnia china & 2,78 & 1,38 \\
14. Asisto a grupos y organizaciones que están compuestas por personas de mi grupo & 2,11 & 1,42 \\
& étnico & 1,03 & 1,08 \\
15. Me siento rechazado por la gente española & 1,25 & 1,15 \\
16. Me siento discriminado por gente de otros grupos étnicos & 0,87 & 1,13 \\
17. Me siento o me he sentido recriminado por gente china cercana a mí por relacionarme & & \\
& con gente española & 39,70 & 19,19 \\
\hline & &
\end{tabular}

Por otro lado, los sujetos muestran un nivel alto en la dimensión Social (ver Tabla 4), con un promedio de 22,44 sobre 28 . Concretamente, presentan un nivel alto de respeto hacia las costumbres y las tradiciones de China y de España, y valoran con niveles altos la importancia que tienen las normas de convivencia de España y que las personas del grupo étnico sepan convivir con otros grupos étnicos (ítems 18-21). Asimismo, se observan valoraciones de nivel medio-alto en la importancia de introducir las normas de otra cultura a personas del grupo étnico y que para sentirse más integrado en la 
sociedad hay que tener una buena conducta hacia los españoles (ítems 22 y 28).

Tabla 4.

Medidas y desviaciones típicas de los ítems de la dimensión Social

\begin{tabular}{|c|c|c|c|}
\hline & & Media & D.T. \\
\hline$\overline{18 .}$ & Respeto las costumbres y las tradiciones de China & 3,52 & 0,74 \\
\hline 19. & Respeto las costumbres y las tradiciones de España & 3,38 & 0,86 \\
\hline 20. & Creo que son importantes las normas de convivencia de España & 3,41 & 0,78 \\
\hline 21. & $\begin{array}{l}\text { Creo que las personas de mi grupo étnico deben saber convivir con otros grupos } \\
\text { étnicos }\end{array}$ & $\mathrm{s}_{3,30}$ & 0,89 \\
\hline 22. & $\begin{array}{l}\text { Considero importante introducir las normas de otra cultura a personas de } \mathrm{mi}_{2} \\
\text { grupo étnico }\end{array}$ & $i_{2,98}$ & 1,00 \\
\hline 23. & $\begin{array}{l}\text { Considero que es importante tener una buena conducta para que la sociedad me } \\
\text { acepte }\end{array}$ & $e_{3,22}$ & 0,95 \\
\hline 24. & $\begin{array}{l}\text { Creo que tener una buena conducta hacia los españoles me hace sentir más } \\
\text { integrado en la sociedad }\end{array}$ & $s_{2,63}$ & 1,14 \\
\hline SO & IAL $(0-28)$ & 22,44 & 6,36 \\
\hline
\end{tabular}

\section{Análisis Diferenciales}

Para llevar a cabo los análisis diferenciales, se tomaron cada uno de los ítems del instrumento y las variables sociodemográficas contempladas en el estudio (ver Tabla 5), aplicando las pruebas estadísticas t de Student y ANOVA de un factor, ambas para grupos independientes.

Los estudios diferenciales en función del Sexo, indican que las mujeres chinas valoran significativamente más alto aspectos referidos con el sentimiento de orgullo por pertenecer a su grupo étnico, con la preferencia de que la pareja sea de etnia china y con la importancia de tener una buena conducta para que sean aceptados por la sociedad (ítems 6, 13 y 23), con un tamaño del efecto pequeño (eta ${ }^{2}$ entre 0,023 y 0,025), según Hair, Anderson, Tathan y Black (2009). En cuanto a los estudios en función de la Edad, el sentimiento de felicidad por ser miembro del grupo étnico y el respeto a las costumbres y tradiciones de España (ítems 5 y 19) es significativamente superior en aquellos sujetos de 14 a 24 años respecto a los de 25 a 35 años, con un tamaño del efecto pequeño (eta ${ }^{2}$ entre 0,021 y $0,029)$. 


\section{Zhou \& Rodríguez-Mantilla-Pertenencia Jóvenes Chinos}

En relación con la variable País de nacimiento, los sujetos nacidos en China presentan niveles significativamente más altos que los nacidos en España u otros países, referidos al aprendizaje sobre los valores o la historia del grupo étnico, a la relación principalmente con chinos, a la asistencia a organizaciones compuestas por chinos o al sentimiento de recriminación por parte de gente china cercana por relacionarse con gente española (ítems 2 , $3,11,14$ y 17), con tamaños del efecto pequeños y moderados (eta ${ }^{2}$ entre 0,032 y 0,088) y el conocer más sobre la historia de China que de España (ítem 4) con un tamaño del efecto grande $\left(\mathrm{eta}^{2}=0,262\right)$.

Tabla 5.

Análisis diferenciales en función de las variables sociodemográficas

\begin{tabular}{|c|c|c|c|c|c|c|c|c|c|c|c|c|c|c|c|c|}
\hline \multirow{2}{*}{$\stackrel{\mathscr{\Xi}}{\Xi}$} & \multicolumn{2}{|l|}{ Edad } & \multicolumn{2}{|l|}{ Sexo } & \multicolumn{2}{|c|}{$\begin{array}{l}\text { País de } \\
\text { nacimiento }\end{array}$} & \multicolumn{2}{|c|}{$\begin{array}{l}\text { Tiempo } \\
\text { España }\end{array}$} & \multicolumn{2}{|c|}{$\begin{array}{l}\text { Nivel } \\
\text { educativo }\end{array}$} & \multicolumn{2}{|c|}{$\begin{array}{l}\text { Ocupación } \\
\text { actual }\end{array}$} & \multicolumn{2}{|c|}{$\begin{array}{l}\text { Amigos } \\
\text { españoles }\end{array}$} & \multicolumn{2}{|c|}{$\begin{array}{l}\text { Amigos } \\
\text { chinos }\end{array}$} \\
\hline & Sig & $\mathrm{Eta}^{2}$ & Sig & $\mathrm{Eta}^{2}$ & Sig & $\mathrm{Eta}^{2}$ & Sig & $\mathrm{Eta}^{2}$ & Sig & $\mathrm{Eta}^{2}$ & Sig & $\mathrm{Eta}^{2}$ & Sig & $\mathrm{Eta}^{2}$ & Sig & $\mathrm{Eta}^{2}$ \\
\hline 2 & & & & & 0,037 & 0,032 & & & & & 0,011 & 0,043 & & & & \\
\hline 3 & & & & & 0,000 & 0,078 & & & & & 0,044 & 0,031 & & & & \\
\hline 4 & & & & & 0,000 & 0,262 & 0,000 & 0,234 & & & & & & & 0,019 & 0,027 \\
\hline 5 & 0,014 & 0,029 & & & & & & & 0,033 & 0,051 & & & & & & \\
\hline 6 & & & 0,031 & 0,023 & & & & & & & & & & & & \\
\hline 7 & & & & & & & & & & & & & & & 0,000 & 0,077 \\
\hline 8 & & & & & & & & & 0,034 & 0,051 & & & & & 0,001 & 0,053 \\
\hline 10 & & & & & & & & & & & & & & & 0,001 & 0,053 \\
\hline 11 & & & & & 0,007 & 0,048 & 0,000 & 0,098 & & & 0,024 & 0,036 & & & 0,005 & 0,038 \\
\hline 12 & & & & & & & & & 0,001 & 0,084 & 0,001 & 0,068 & & & & \\
\hline 13 & & & 0,024 & 0,025 & & & 0,032 & 0,043 & & & & & & & & \\
\hline 14 & & & & & 0,000 & 0,088 & 0,008 & 0,057 & 0,017 & 0,058 & 0,018 & 0,039 & & & 0,001 & 0,057 \\
\hline 15 & & & & & & & & & & & & & 0,009 & 0,033 & & \\
\hline 16 & & & & & & & & & & & 0,026 & 0,036 & 0,001 & 0,056 & & \\
\hline 17 & & & & & 0,020 & 0,038 & 0,025 & 0,045 & 0,004 & 0,074 & 0,000 & 0,094 & 0,007 & 0,035 & & \\
\hline 18 & & & & & & & & & & & 0,011 & 0,044 & & & 0,004 & 0,041 \\
\hline 19 & 0,040 & 0,021 & & & & & & & & & 0,002 & 0,060 & & & & \\
\hline 20 & & & & & & & & & & & & & 0,000 & 0,060 & & \\
\hline 21 & & & & & & & 0,013 & 0,052 & 0,025 & 0,054 & 0,005 & 0,051 & 0,004 & 0,041 & & \\
\hline 23 & & & 0,034 & 0,022 & & & & & & & & & & & & \\
\hline
\end{tabular}


Respecto al tiempo de residencia en España, los análisis muestran que los sujetos que llevan 10 años o menos en España, puntúan con niveles significativamente más altos en la relación fundamentalmente con chinos, la asistencia a grupos y organizaciones compuestas por personas del mismo grupo étnico, la recriminación por gente china cercana por relacionarse con españoles (ítems 11,14 y 17, con un tamaño del efecto moderado, eta ${ }^{2}$ entre $0,045$ y 0,098$)$ y el conocimiento sobre las historia de China más que de España (ítem 4) con un tamaño del efecto grande (eta $\left.{ }^{2}=0,234\right)$. Por otro lado, los sujetos que llevan 11 años o más en España, valoran con puntuaciones significativamente más altas aspectos relacionados con la preferencia de que la pareja sea de etnia china y con la creencia de que las personas del mismo grupo étnico deban saber convivir con otros grupos étnicos (ítems 13 y 21), con un tamaño del efecto moderado (eta ${ }^{2}$ entre 0,043 y 0,052).

En función de la variable Nivel Educativo, los resultados muestran que los sujetos que poseen estudios de Educación Secundaria y de nivel superior (FP o Universitarios), perciben con un nivel significativamente mayor la importancia de mantener relaciones con españoles y la asistencia a organizaciones compuestas por personas del mismo grupo étnico (ítems 12 y 14), con respecto a los sujetos que tienen estudios de Primaria o sin estudios, con un tamaño del efecto moderado (eta ${ }^{2}$ entre 0,058 y 0,084 ). Por su parte, la felicidad que provoca formar parte del grupo étnico, el sentimiento de sentirse apoyados por chinos cuando tienen problemas, el sentimiento de recriminación por parte de gente china cercana por relacionarse con gente española y la creencia de que las personas de su grupo étnico deban saber convivir con otros grupos étnicos (ítems 5, 8, 17 y 21), son valorados de forma significativamente mayor por los sujetos con estudios de Primaria o sin estudios, con un tamaño del efecto moderado (eta ${ }^{2}$ entre 0,051 y 0,074).

Respecto a la Ocupación actual de los sujetos, los análisis muestran que los estudiantes valoran de forma significativamente superior la relación principal con chinos, la búsqueda de relaciones con españoles, la importancia de las normas de convivencia de España y de tener una buena conducta para que la sociedad les acepte (ítems 11, 12, 20 y 23), respecto de los que tienen un puesto de trabajo, con tamaños del efecto pequeños y moderados ( eta $^{2}$ entre 0,036 y 0,060). Mientras tanto, los que no trabajan ni 
estudian, presentan niveles significativamente más altos que los otros en relación al sentimiento de recriminación por gente china cercana por relacionarse con gente española y el respeto a las costumbres y tradiciones de China (ítems 17 y 18), con un tamaño del efecto moderado (eta ${ }^{2}$ entre 0,044 y 0,094).

En función de si los sujetos tienen amigos españoles o no, los resultados muestran que estos últimos puntúan con niveles significativamente más altos aspectos relacionados con el sentimiento de rechazo por gente española, de discriminación por gente de otros grupos étnicos y de recriminación por gente china cercana por relacionarse con gente española (ítems 15, 16 y 17). Por su parte, los que sí tienen amigos españoles, valoran con puntuaciones significativamente más altas aspectos relacionados con la creencia de la importancia de las normas de convivencia de España y de que las personas del propio grupo étnico sepan convivir con otros grupos étnicos (ítems 20 y 21). Igualmente, los que tienen amigos chinos valoran con puntuaciones significativamente más altas, aspectos relacionados con el conocimiento de la historia de China y el respeto de sus costumbres y tradiciones, el sentimiento de una fuerte unión con el propio grupo étnico, apoyado por ellos cuando tienen problemas, sentirse mejor y relacionarse principalmente con ellos (ítems 4, 7, 8, 10, 11, 14 y 18), con tamaños del efecto pequeños y moderados ( eta $^{2}$ entre 0,027 y 0,077 ).

\section{Análisis Clúster}

Para explorar la existencia de distintos posibles perfiles de jóvenes chinos, vinculado a los indicadores analizados en el presente estudio, se realizó un análisis clúster o de conglomerados por el procedimiento de $\mathrm{k}$ medias, obteniendo como satisfactoria la configuración de 3 clústeres.

Respecto al número de variables, inicialmente se incluyó el conjunto total de ítems para ver cómo se comportaba cada uno de ellos. Sin embargo, los resultados no mostraron variabilidad significativa entre los distintos clústeres en los ítems 3, 9, 12 y 24, por lo que fueron eliminados de la interpretación los mismos al resultar no discriminantes. Los ítems finalmente utilizados se muestran en la Tabla 6. 
Tabla 6.

Centros de los conglomerados finales por ítems

\begin{tabular}{|c|c|c|c|}
\hline \multirow{2}{*}{$\begin{array}{c}\text { h } \\
\text { Ítems }\end{array}$} & \multicolumn{3}{|c|}{ Conglomerados } \\
\hline & 1 & 2 & 3 \\
\hline 1 & 2,33 & 3,07 & 3,44 \\
\hline 2 & 2,00 & 2,19 & 3,06 \\
\hline 4 & 1,47 & 0,55 & 2,18 \\
\hline 5 & 2,40 & 2,75 & 3,65 \\
\hline 6 & 2,40 & 2,91 & 3,69 \\
\hline 7 & 1,80 & 2,31 & 3,41 \\
\hline 8 & 2,00 & 2,12 & 3,36 \\
\hline 10 & 1,87 & 1,66 & 2,92 \\
\hline 11 & 2,27 & 0,54 & 2,11 \\
\hline 13 & 2,13 & 1,45 & 3,59 \\
\hline 14 & 1,67 & 0,97 & 2,79 \\
\hline 15 & 2,07 & 0,57 & 1,15 \\
\hline 16 & 2,07 & 0,85 & 1,37 \\
\hline 17 & 2,33 & 0,75 & 0,76 \\
\hline 18 & 2,27 & 3,37 & 3,76 \\
\hline 19 & 2,20 & 3,30 & 3,56 \\
\hline 20 & 2,33 & 3,48 & 3,51 \\
\hline 21 & 2,00 & 3,51 & 3,35 \\
\hline 22 & 2,27 & 2,97 & 3,07 \\
\hline 23 & 2,27 & 3,13 & 3,38 \\
\hline
\end{tabular}

A partir de los resultados obtenidos se han podido definir cada uno de los clústeres del siguiente modo: 


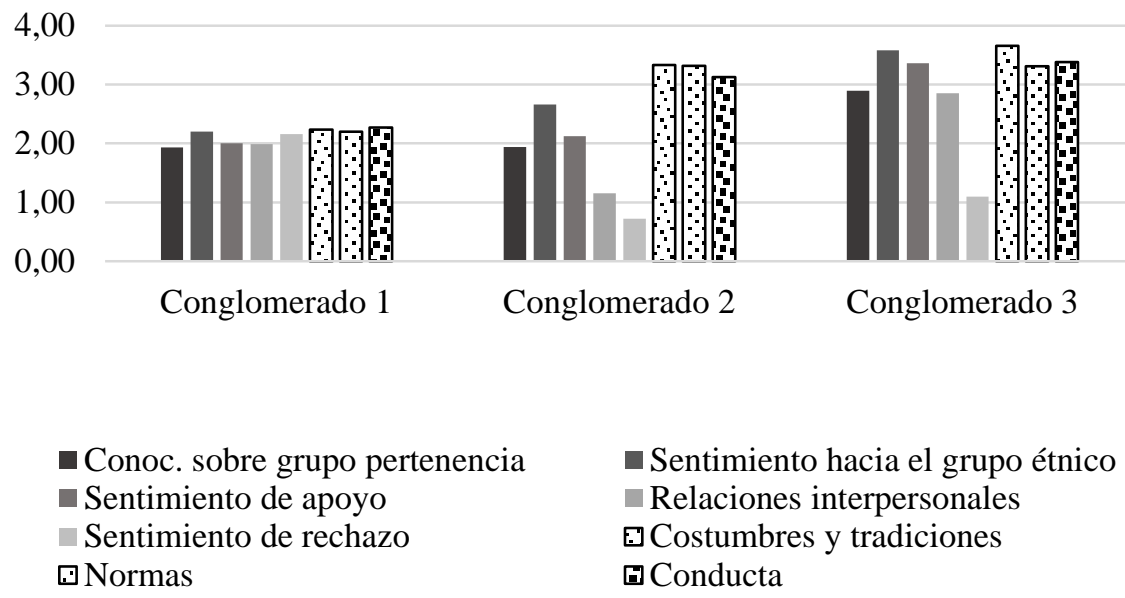

Figura 1. Representación de los clústeres en función de las puntuaciones medias por subdimensiones

- El conglomerado 1, formado por el 7,32\% de la muestra, corresponde a sujetos de etnia china con puntuaciones de tipo medio-bajo en la dimensión Cognitivo-Afectiva (representada con texturas lisas en el Gráfico 1) y en la dimensión Social (con texturas rugosas en el Gráfico 1). De forma específica, analizando los resultados de la Tabla 6, encontramos que los sujetos presentan puntuaciones medio-bajas en las subdimensiones referidas al conocimiento sobre el grupo de pertenencia (ítems 1, 2 y 4), el sentimiento de apoyo (ítem 8) y las relaciones interpersonales (ítems 10, 11, 13 y 14). En el resto de subdimensiones, los sujetos chinos de este conglomerado presentan puntuaciones de nivel medio en lo referido al sentimiento hacia el grupo étnico (ítems 5-7), el sentimiento de rechazo (ítems 15-17), las costumbres y tradiciones (ítems 18-19), las normas (ítems 20-22) y la conducta (ítem 23) (ver Tabla 6).

- El conglomerado 2 está formado por el 32,68\% de la muestra. Este grupo se caracteriza por poseer puntuaciones muy altas en la dimensión Social (en las subdimensiones costumbres, tradiciones, normas y conductas sociales -ítems 17-23-) y puntuaciones medias en aspectos 
relativos al conocimiento y sentimiento hacia el grupo étnico (ítems 1, 2 y 4-8). Por otro lado, los sujetos chinos de este conglomerado presentan puntuaciones bajas o muy bajas en las subdimensiones sobre las relaciones interpersonales y el sentimiento de rechazo (ítems 10-14 y 15-17).

- El conglomerado 3, formado por el $60 \%$ de la muestra, se configura por sujetos con puntuaciones altas o muy altas en las dos grandes dimensiones analizadas (Cognitivo-Afectiva y Social). En concreto, los sujetos de etnia china de este conglomerado presentan valoraciones muy altas en las subdimensiones relacionadas con el conocimiento sobre el grupo de pertenencia (ítems 1, 2 y 4), el sentimiento hacia el grupo étnico (ítems 5-7), el sentimiento de apoyo (ítem 8) y las costumbres, tradiciones, normas y conductas sociales (ítems 18-23). Por otro lado, estos sujetos presentan valoraciones de nivel medio en la subdimensión relacionada con las relaciones interpersonales (ítems 10, 11, 13 y 14) y de nivel bajo en el sentimiento de rechazo (ítems 15-17).

\section{Discusión y Conclusiones}

El presente trabajo ha permitido dar respuesta a los objetivos propuestos relacionados con el nivel de sentimiento de pertenencia que presentan los jóvenes chinos de la Comunidad de Madrid, encontrando que los sujetos participantes en el estudio perciben, en términos generales, un nivel medioalto de sentimiento de pertenencia hacia el grupo étnico al que pertenecen.

De igual modo, el trabajo realizado permite obtener una serie de conclusiones, las cuales se exponen a continuación:

- En relación con la dimensión Cognitivo-Afectiva de los sujetos de la muestra participante, la mayoría de ellos comprenden lo que significa e implica formar parte de su propio grupo étnico, se sienten felices y orgullosos del grupo étnico al que pertenecen. Además, no se sienten rechazados por la gente española o de otros grupos étnicos y no procuran relacionarse principalmente con chinos. No obstante, manifiestan conocer poco sobre la historia de China, siendo este un elemento clave en el sentimiento de pertenencia hacia el grupo étnico, y relacionado de forma directa con un mayor nivel de participación y con mejores resultados escolares (Oñate, Aretxabala, Armendari \& Oiarzabal, 2012). 


\section{Zhou \& Rodríguez-Mantilla-Pertenencia Jóvenes Chinos}

- Respecto a la dimensión Social, la mayoría de los jóvenes chinos participantes, consideran importante el respeto hacia las costumbres y tradiciones tanto de China como de España. En este sentido, autores como Martínez, Paterna y Goveia (2006) señalan la importancia que tiene para una persona mantener sus costumbres y valores tradicionales cuando realiza el proceso de aculturación, dado que le aporta un sentimiento de continuidad en la vida a la vez que le proporciona contacto con un grupo con la misma forma de ver el mundo. Por otro lado, los jóvenes chinos manifiestan la importancia del cumplimento de las normas de convivencia y el desarrollo de una buena conducta para que la sociedad les acepte. En este sentido, siendo el respeto un componente importante para los jóvenes chinos, autores como Chao \& Tseng (2002), afirman la importancia de la familia en el desarrollo de valores como el respeto y la educación en las relaciones sociales, resaltando la interdependencia entre los miembros de la familia como una característica general de la cultura asiática.

Por otro lado, los estudios diferenciales llevados a cabo han permitido obtener las siguientes evidencias:

- Las jóvenes chinas valoran significativamente más alto que los varones aspectos referidos con el sentimiento de orgullo y la importancia de tener una buena conducta para que sean aceptados por la sociedad. Estos resultados pueden estar relacionados con el hecho de que, en términos generales, son las mujeres las que valoran con un mayor interés el desarrollo personal y la calidad de las relaciones sociales (según Rodríguez, Matud \& Álvarez, 2017; Zubieta, Muratori \& Fernández, 2012).

- En función de la edad, los sujetos más jóvenes (de 14 a 24 años) manifiestan mayor felicidad por ser miembro del grupo étnico, con respecto a los sujetos de más edad (de 25 a 35 años). En esta misma línea, autores como Auné, Abal y Attorresi (2017) y Beytia (2016) afirman que la edad es un factor directo asociado con el nivel de felicidad y, a su vez, con la calidad de los lazos familiares.

- Los sujetos que han nacido en China y que llevan menos de 10 años en España, coinciden en que conocen la historia de China más que de España, que se relacionan principalmente con chinos o que asisten a organizaciones compuestas por chinos. Esto puede ser debido a que han 
pasado menos tiempo en un país con unas tradiciones y costumbres totalmente diferentes a las natales, provocando una difícil integración. Autores como De Lizarrondo Artola, Rinken, Márquez y Godenau (2016) y Pascual (2013) afirman que los inmigrantes aparecen en la sociedad de acogida como un grupo social bien diferenciado, de manera que parece difícil una integración completa, debido a la coexistencia de grupos con culturas diferentes en un mismo espacio.

- En función del nivel educativo, los sujetos que tienen estudios de Primaria o que no tienen estudios, presentan un mayor sentimiento de felicidad por formar parte del grupo étnico. En este sentido, autores como Beytia (2016) y Marmot y Wilkinson (2006) afirma que el nivel educativo es un factor indirecto asociado con el nivel de felicidad, y que para entender estos niveles es necesario analizar la calidad de los lazos familiares. Además, la relación de la felicidad con el nivel educativo es difícil de asociar, ya que generalmente los bajos niveles de educación formal se relacionan con una menor esperanza de vida y menor bienestar.

- Los jóvenes chinos que no tienen amigos españoles presentan un mayor sentimiento de rechazo por gente española, de discriminación por gente de otros grupos étnicos y de recriminación por gente china cercana por relacionarse con gente española. Sin embargo, la mayoría de los sujetos, que tienen amigos de ambas nacionalidades, consideran igual de importante mantener relaciones de amistad tanto con las personas de sus países como con los españoles. En este sentido, autores como Mercado Maldonado y Hernández Oliva (2010) afirman que los individuos examinan mejor la pertenencia al grupo étnico cuando se relacionan con miembros de grupos diferentes al suyo. No obstante, los resultados aquí obtenidos han de ser tomados en cuenta con precaución dado el importante desequilibrio en la muestra de sujetos que sí y no manifiestan tener amigos españoles.

Finalmente, el análisis de conglomerados ha permitido definir tres claros perfiles de sujetos de etnia china en función de su sentimiento de pertenencia:

- Los sujetos del perfil 1, que representa una minoría de la muestra, presentan un nivel medio de sentimiento de pertenencia. Así, estos sujetos, manifiestan valores moderados en lo relativo al conocimiento de 
lo que significa e implica formar parte de un grupo étnico, su relación social principalmente con chinos, el sentimiento de felicidad y de orgullo por formar parte del grupo étnico, el respeto de las normas y costumbres de China y de España y la importancia de las normas y las conductas.

- Los sujetos del perfil 2 presentan un nivel medio alto de sentimiento de pertenencia. En concreto, los sujetos de este conglomerado presentan niveles altos en la dimensión Social (en aspectos relacionados con las costumbres, tradiciones, normas y conductas) y niveles bajos en el sentimiento de rechazo, es decir, no se sienten rechazados por españoles $\mathrm{u}$ otros grupos étnicos ni recriminados por gente cercana por relacionarse con gente española. Por otro lado, presentan niveles medios en aspectos relacionados con el conocimiento y el sentimiento hacia el grupo étnico.

- Los sujetos del perfil 3, correspondientes al 60\% de la muestra de estudio, presentan un nivel alto de sentimiento de pertenencia, manifestando niveles altos en todas las dimensiones evaluadas y un sentimiento muy bajo de rechazo.

De este modo, y como se ha señalado anteriormente, el trabajo realizado ha permitido alcanzar los objetivos inicialmente propuestos aportando información relevante en el campo de conocimiento estudiado, si bien cabe señalar como limitación el tamaño de la muestra obtenida, debido a las dificultades que ha supuesto acceder a la población de estudio y lograr la participación de los individuos. No obstante, este mismo hecho, junto a los resultados aquí presentados, pueden servir para profundizar en estudios posteriores sobre aspectos relacionados con la identificación de las causas del nivel del sentimiento de pertenencia alcanzado por los jóvenes chinos desde un enfoque cualitativo (aplicando entrevistas, realizando pequeños grupos de discusión, etc.) o el estudio de la relación entre el sentimiento de pertenencia y el rendimiento académico de dichos jóvenes.

\section{Referencias}

Antolín, J. B. (2010). Comunidades asiáticas en España: movilidad transnacional en un territorio de frontera. Revista CIDOB d'afers internacionals, 15-37. 
Aparicio, R., \& Portes, A. (2014). Crecer en España: La integración de los hijos de inmigrantes. Obra Social" La Caixa".

Arenas, P., \& Urzúa, A. (2016). Estrategias de aculturación e identidad étnica: un estudio en migrantes sursur en el norte de Chile. Universitas Psychologica, 15(1), 117-128.

Auné, S. E., Abal, F. J. P., \& Attorresi, H. F. (2017). Efectos del género y la educación en la felicidad en adultos argentinos. Liberabit, 23(2), 169188.

Barquín, A. (2009). ¿De dónde son los hijos de los inmigrantes? La construcción de la identidad y la escuela. Educar, 44, 81-96.

Bartolomé, M., Cabrera, F., Espín, J. V., Del Campo, J., Marín, M. A., Rodríguez, M., ... y Sabariego, M. (2000). La construcción de la identidad en contextos multiculturales. Madrid: CIDE.

Beytía, P. (2016). The singularity of Latin American patterns of happiness. En Handbook of happiness research in Latin America (pp. 17-29). Dordrecht, Holanda: Springer.

Brea, L. (2014). Factores determinantes del sentido de pertenencia de los estudiantes de arquitectura de la Pontificia Universidad Católica Madre y Maestra, Campus Santo Tomás de Aquino (tesis doctoral). Universidad de Murcia, España.

Cameron, J. E. (2004). A three-factor model of social identity. Self and identity, 3(3), 239-262. doi: 10.1080/13576500444000047

Chao, R. K., \& Tseng, V. (2002). Parenting of Asians. En M. H. Bornstein (Ed), Handbook of parenting: Vol. 4. Social conditions and applied parenting ( $2^{\mathrm{a}}$ ed., pp. 59-93). Mahwah, NJ: Erlbaum.

Clemente, M., Espinosa, P., \& Antón, M. F. (2013). La integración de los inmigrantes a través de la comunicación. BARATARIA. Revista Castellano-Manchega de Ciencias sociales, 16, 83-96.

Dávila de León, C., \& Jiménez García, G. (2014). Sentido de pertenencia y compromiso organizacional: predicción del bienestar. Revista de Psicología (PUCP), 32(2), 271-302.

De Lizarrondo Artola, A. M., Rinken, S., Márquez, G. M., \& Godenau, D. (2016). La integración del colectivo inmigrante en las regiones españolas. Papers: revista de sociología, 101(3), 289-313.

Etxeberría, F., \& Elosegui, K. (2010). Integración del alumnado inmigrante: obstáculos y propuestas. Revista Española de Educación Comparada, 


\section{Zhou \& Rodríguez-Mantilla-Pertenencia Jóvenes Chinos}

$16,235-264$.

Fenster, T. (2005). Gender and the city: the different formations of belonging. En L. N. Seager, A Companion to Feminist Geography Routledge (pp. 242-256). Malden, Massachuset, USA: Blacwell Publishing Ltd.

Giró, J. (2010). Identidad étnica, adolescencia y aculturación. En Bernuz, $M^{a}$. y Susín, R., (coords.) Seguridad, excepción y nuevas realidades jurídicas (pp.161-176). Granada: Comares.

Gutiérrez Sastre, M. (2014). Emprendimiento familiar inmigrante: el caso de las mujeres chinas en España. Revista de Empresa Familiar, 4(2), 70.

Hair, J., Anderson, R., Tathan, R. y Black, W. (2009). Análisis multivariante. Madrid: Pearson.

Hayes, N. (2013). Los estereotipos culturales como obstáculo para la convivencia en la escuela inclusiva. Tejuelo: Didáctica de la Lengua y la Literatura. Educación, (18), 101-114.

Huang, Y. T. (2015). Las dimensiones culturales aplicadas a la enseñanza/aprendizaje del ELE: un análisis de caso entre la cultura española y la cultura china. marcoELE. Revista de Didáctica Español Lengua Extranjera, 20.

Huerta Orozco, A. (2018). El sentido de pertenencia y la identidad como determinante de la conducta, una perspectiva desde el pensamiento complejo. IE Revista de investigación educativa de la REDIECH, 9(16), 83-97.

Instituto Nacional de Estadística (2018). Recuperado de http://www.ine.es/

Kiang, L., Perreira, K. M., \& Fuligni, A. J. (2011). Ethnic label use in adolescents from traditional and non-traditional immigrant communities. Journal of Youth and Adolescence, 40, 719-729.

Lara, L. (2017). Adolescentes latinoamericanos en España: Aculturación, autonomía conductual, conflictos familiares y bienestar subjetivo. Universitas Psychologica, 16(2), 26-36.

Lara, L. \& Martínez-Molina, A. (2016). Validación de la Escala de Identidad Étnica Multigrupo-Revisada en adolescentes inmigrantes y autóctonos residentes en España. Revista Latinoamericana de Ciencias Sociales, Niñez y Juventud, 14(1), pp. 591-601.

Maillard, C., Ochoa, G., \& Valdivia, A. (2018). Experiencia educativa e identidades étnicas en estudiantes universitarias indígenas de la 
Región Metropolitana. Calidad en la educación, 28, 176-201. Marmot, M., \& Wilkinson, R. G. (2006). Social determinants of health. Oxford, Inglaterra: Oxford University Press.

Martínez, A. G. (2003). Identidad y cultura: Efectos en la educación intercultural. Pedagogía social: revista interuniversitaria, 10, 253264.

Martínez, M. C., Paterna, C., \& Goveia, V. (2006). Relevancia del modelo dual de valores en relación con el prejuicio y la intención de contacto hacia exogrupos. Anales de Psicología, 22, 243-250.

Mercado Maldonado, A., \& Hernández Oliva, A.V. (2010). El proceso de construcción de la identidad colectiva. Convergencia, 17(53), 229251.

Merenstein, B. (2001). La construcción de identidad en las minorías: acerca de su importancia y sus consecuencias teóricas. Araucaria: Revista Iberoamericana de filosofía, política y humanidades, 6, 96-112.

Mieles-Barrera, M. D., \& Vesga, M. C. G. (2010). Apuntes sobre socialización infantil y construcción de identidad en ambientes multiculturales. Revista Latinoamericana de Ciencias Sociales, Niñez y Juventud, 8(2), 809-819.

Molano, O. L. (2007). Identidad cultural un concepto que evoluciona. Opera, 7, 69-84.

Nieto, G. (2003). La inmigración china en España. Definiciones y actuaciones sobre integración social. Revista CIDOB d'afers internacionals, 167-189.

Olivencia, J. J. L. (2011). Participación de las familias inmigrantes y educación intercultural en la escuela. Contextos educativos: Revista de educación, 14, 119-134.

Oñate, C. M., Aretxabala, E., Armendariz, A. I., \& Oiarzabal, P. J. (2012). El concepto de Pertenencia (Belonging) como marco analítico para la investigación sobre la juventud de origen inmigrante. Anuario de acción humanitaria y derechos humanos. Yearbook of humanitarian action and human rights, 10, 101-112.

Pascual, M. S. (2013). La integración sociolingüística de la inmigración hispana en España: lengua, percepción e identidad social. Lengua y migración/Language and Migration, 5(2), 91-110.

Pérez, M. I. S. (2016). Estudio de Casos sobre el Grado de Intervención de la 
Milics en el Ámbito de la Salud Sexual y Reproductiva: Usuarias de Origen Chino. FITISPos International Journal, 3, 184-194.

Phinney, J. S. (2003). Ethnic identity and accculturaction. En K. M. Chung, P. Balls Organista, \& G. Marín (Eds.) Acculturation: Advances in theory, measurement, and applied research (pp. 63-81). Washington, DC: American Psychological Association.

Robles-Llana, P. (2018). Cultural Identities of Children of Chinese Migrants in Spain: A Critical Evaluation of the Category 1.5

Generation. Identity, 18(2), 124-140. doi:

10.1080/15283488.2018.1447481

Rodríguez Belmaresa, P., Matud Aznar, M. P., \& Álvarez Bermúdez, J. (2017). Género y calidad de vida en la adolescencia. Journal of Behavior, Health \& Social Issues, 9(2), 89-98. doi:

10.1016/j.jbhsi.2017.11.001

Rodríguez-Wangüemert, C., Rodríguez-Breijo, V., \& Pestano-Rodríguez, J.

M. (2019). The framing of China on Spanish

television. Communication \& Society, 32(3). doi:

10.15581/003.32.3.123-137

Sáiz López, A. (2015). Mujeres y sociedad civil en la diáspora china: el caso español. Inter Asia Papers, 47, 1-35.

Smith Castro, V. (2002). La Escala de Identidad Etnica Multigrupo (EIEM) en el contexto costarricense 1. Actualidades en psicología, 18(105), 47-67.

Strayhorn, T. L. (2012). College students' sense of belonging: A key to educational success for all students. Routledge.

Terrén, E. (2011). Identidades desterritorializadas. El sentimiento de pertenencia nacional entre los adolescentes de familias inmigradas. Papers. Revista de Sociologia, 96(1), 97-116.

Torruella, I. M., \& López, A. S. (2017). Género, movilidad e intersecciones generacionales en el espacio transnacional chino. RES. Revista Española de Sociología, 26, 385-397.

Vargas Alfaro, A. T. (2002). Identidad y sentido de pertenencia. Una mirada desde la cotidianeidad. Centro Prov. de Cultura Comunitaria. Ciudad de La Habana, Cuba.

Vega, H. F. (2011). Identidad e inmigrantes: casos particulares, procesos generales. In Actas del I Congreso Internacional sobre Migraciones en 
Andalucía (pp. 1639-1648). Instituo de Migraciones.

Wang, L., \& Lorenzo, M. T. C. (2019). Factores y estrategias del aprendiz preadolescente en chino como lengua extranjera. Estudio de caso en España. Onomázein: Revista de lingüística, filología y traducción de la Pontificia Universidad Católica de Chile, 43, 158-175.

Zubieta, E., Muratori, M. \& Fernández, O. (2012). Bienestar subjetivo y psicosocial: explorando diferencias de género. Salud \& Sociedad: investigaciones en psicología de la salud y psicología social, 3(1), 6676.

Weina Zhou is a Master Student at the Complutense University of Madrid, Spain

Jesús Miguel Rodríguez-Mantilla is profesor at the Complutense University of Madrid, Spain

Contact Address: weinazho@ucm.es 